\title{
NON-MEDICAL MASKS: OPPORTUNITIES FOR STANDARDS Education ANd Online Design ProJects
}

\author{
David Torvi \\ Department of Mechanical Engineering \\ University of Saskatchewan \\ david.torvi@usask.ca
}

\begin{abstract}
While engineering students gain some experience in the use of codes and standards, some may not be exposed to the process used to develop standards, or the history of individual standards. A number of resources on standard development are available to instructors, and knowing the history of a standard will aid in understanding its potential limitations when used in design. This paper will outline how the process of developing standard test methods for non-medical masks during the COVID-19 pandemic can be used as a case study in design courses. Potential online projects and assignments related to testing of these masks are described, including considerations of material performance, comfort and functional fit, along with examples of analysis that students could perform. Sample fabric tests that use readily-available supplies to measure water resistance are described to illustrate how assignments and projects could be completed by students remotely in an online course.
\end{abstract}

Keywords: design, standards, masks, face coverings, protective clothing, PPE, remote learning, COVID-19.

\section{INTRODUCTION}

Non-medical masks, or barrier face coverings, have been a major focus during the COVID-19 pandemic, given shortages in personal protection equipment (PPE), such as N95 respirators and surgical masks. Since March, 2020, options for fabric masks have significantly increased, including hospitals and fabric retailers making patterns and instructions available for those who choose to make their own masks (e.g., $[20,24,26])$. While requirements for PPE are outlined in standards, such as 42 CFR Part 84 for respirators [1] and ASTM F2100 for surgical masks [6], governments and standard development organizations have also begun to develop guidelines and standards for nonmedical masks. In the early days of the pandemic this included advice on how to manufacturer your own barrier face coverings using fabrics that would be easily available in the home, such as t-shirts, given that there was a shortage of available products for consumers [19].

CEEA-ACEG21; Paper 024

University of Prince Edward Island; June 21 - 23, 2021- 1 of 7 -
As more commercial products have become available, guidance has been provided on how to select a mask. For example, at the time of this writing, Health Canada recommends that a non-medical mask be constructed using at least two layers of cotton or other tightly woven, breathable fabric with a filter (e.g., polypropylene) in between the two layers [19]. Besides using a mask made of at least two layers, the Center for Disease Control (CDC) in the U.S. provides information on improving the fit and filtration of these masks, including using a mask with a nose wire or a mask fitter, knotting and tucking the ear loops on a medical procedure mask, and wearing a medical procedure mask underneath a cloth mask [13].

Standard development organizations have also begun to develop guidelines and standards for non-medical masks (e.g., AATCC M14 [2], AFNOR Spec S76-001 [4], CWA 17553 [15]). In February, 2021, the American Society for Testing and Materials (ASTM) approved F3502, a specification for barrier face coverings [9]. This document includes specifications which evaluate source control provided by a product, as well as the potential for the product to provide some degree of particulate filtration. ASTM F3502 includes requirements for design, performance, labeling, user instruments and other factors. Products are evaluated for sub-micron particle filtration efficiency and air flow resistance (which measures breathability) using tests that are based on 42 CFR Part 84 [1], and a classification system for products is described based on minimum performance requirements.

Given their high visibility and the fact that the use of these products has been mandated since the fall of 2020, non-medical masks can be used in design courses as an example of the development and use of standards. The increased recognition of the need for standards for these products and subsequent efforts by ASTM and other organizations can be used to illustrate the standards development process. Development and application of standards requires an understanding of the end use of the product, which aspects of performance should be evaluated, and the relationship between test conditions and the hazard that is being simulated, all of which have been 
topics of much research and discussion. Consideration of different aspects of performance can also be illustrated, including trade-offs between filtration and breathability.

This paper will first outline some resources that are available for standards education, and describe a lecture on codes and standards that is given in a second year mechanical engineering design course. A case study on existing standards for respirators, surgical masks and other PPE, and the development of new standards for nonmedical masks is then described. Potential online assignments, labs and design projects related to the development of test standards are then discussed. One fabric test that uses readily-available equipment in the home to measure water resistance is described as an example of how this course work could be completed by students remotely.

\section{STANDARDS EDUCATION}

Engineering students gain some experience in the use of codes and standards, which may include lectures on codes and standards, calculations based on building codes or other regulations, or requirements to identify relevant standards in design reports. However, students may not necessarily be exposed to the process used to develop standards, or the history of individual standards that are important to their discipline. Knowing this process and history helps to understand potential limitations of standards in design. The standard development process can be outlined in lectures within design courses and illustrated using case studies, which discuss the history, strengths and limitations of particular standards.

\subsection{Lectures on Codes and Standards}

The author has provided a lecture in a second-year mechanical engineering design project course (ME 229) for more than 10 years to introduce students to the importance of codes and standards in design. The development and use of codes and standards is also a prominent feature in undergraduate (ME 478) and graduate (ME 868) fire protection engineering courses the author teaches. In the ME 229 lecture, examples of standards that are important to mechanical engineering design are provided, including those dealing with pressure vessels, building systems, materials, consumer products and industrial processes. The standards development process is described, with emphasis on the process used to develop ASTM standards. Limitations of standards is discussed, along with certification marks. The National Building and Fire Codes are discussed, including their roles as model codes for provincial and territorial governments when they develop their building regulations. This discussion of building and fire codes is important as mechanical engineering students may not get the same level of exposure to these documents as those in civil engineering and other disciplines. Examples in the lecture are typically taken from areas in which the author participates in the development of standards, including fire safety and protective clothing. Students complete a quiz, which includes questions designed to have them search online for standards for a particular product.

A number of sources of information are available to support standards education. For example, ASTM's website [11] includes a section for students and professors, which links standards education to the lifelong learning graduate attribute [12]. Modules that can be included in lectures in design and other courses are included, along with collections of standards for different engineering disciplines, and case studies and articles highlighting the use of standards in particular industries. Many Canadian university libraries have a subscription to ASTM Compass [10], which includes not only access to standards, but research articles that are published in ASTM journals and conference proceedings. Many of the research articles describe the development of test methods that later go on to become the basis for ASTM and other standards. In one of the author's graduate courses, students are expected to select a standard test of interest to their research or professional interests and explain the history of the standard, including the motivation for its development (e.g., many fire tests were developed in response to a series of fire tragedies). They are also asked to describe any limitations, including how well the test simulates expected conditions in fires of today and evaluates new materials.

This lecture on codes and standards addresses a number of the ME 229 learning outcomes. The engineer's role in society as it applies to health and safety is explained. The role of objectives and constraints, which are needed for a clear problem definition, are demonstrated. Ways in which applicable codes and standards are identified and applied to the design problem are discussed, along with the use of codes and standards in evaluating risks of design alternatives.

One challenge with teaching standards in design courses is that students, especially early in their program, may not have had much experience with materials or products that they will be using in the designs that they will be engaged in as professionals. For instance, while all students in this second-year course will have some knowledge of buildings, few would be expected to have detailed knowledge of wall or floor construction, let alone have built a wall or floor themselves. Therefore, it is preferable to include case studies involving products students are familiar with, especially if they can identify aspects of performance and other criteria that they would use to evaluate products for purchase and use. Given the extent to which these products have been used by students over the last several months, a case study involving non-medical masks was selected this year. 


\subsection{Non-medical Mask Case Study}

In the case study portion of the lecture, the need for standards for non-medical masks was first illustrated. This included a reminder of the lack of consumer products during the spring and early summer of 2020. This was followed by a rapid increase in product availability in the fall of 2020, at the same time that jurisdictions were implementing mask mandates in public places. For example, a New York Times article in December, 2020 on the development of the ASTM F3502 standard estimated that there were more than 100,000 types of masks for sale at that time [21]. It is also difficult for consumers to determine the effectiveness of candidate masks they may be considering for purchase, as product packaging and labeling will typically only include information on the fibre content of the fabrics used to manufacture the masks.

Precise terminology is important in codes and standards, especially as these may be used as legal documents. While many products are commonly referred to as face masks, a number of terms were introduced, each of which has a specific meaning to regulators. For example, while disposable masks are often referred to as medical masks by the public, many of the products currently being sold are not certified to any standard (this may be noted on the packaging, but not evident on an individual product). Respirators and surgical masks are defined, along with Canadian and U.S. regulations and relevant standards for these products. In order to address the need for medical mask and respirators, Health Canada has developed methods to expedite access to these products, including allowing products that meet comparable standards to those found in existing regulations for products [18]. This development was discussed with students, along with the larger issues of evaluating products for use in design that have been tested to different standards, and the impact of standards on international trade.

One issue that has made it difficult to compare products or materials for non-medical masks is the range of test conditions that have been used to evaluate performance in the large number of research papers and other publications that have emerged during the pandemic. One example is the differences between challenges in standard tests used to assess submicron particulate filtration efficiency $(\mathrm{NaCl}$ aerosol particles with a mass median aerodynamic diameter of $0.3 \mu \mathrm{m})$ [1] and bacterial filtration efficiency (mean particle size of $3.0 \mu \mathrm{m}$ ) [7]. It can be noted to students that this is also an issue when comparing filters used in heating, ventilating and air conditioning systems that are tested using different international standards [25]. Another issue is the range of information needed to characterize a particular fabric beyond simply stating the fiber content, and the fact that this additional information may not be readily available for a particular fabric or product.
While standard tests are performed in laboratories to evaluate different aspects of performance, this may not be completely indicative of that product's performance in the field. In the case of masks, fit plays a key role in protection and comfort, and standard tests that are used in fit testing respirators were outlined [14]. The importance of communicating the limitations of methods used to evaluate performance is emphasized, along with the potential for end users to incorrectly use test results as a means of predicting the actual protection a product will provide in a specific situation. To further illustrate this, examples from protective clothing tests, where products are ranked on the basis of predicted skin burn damage, were provided.

Potential selection criteria for barrier face coverings were outlined, including protection, comfort, functional fit, durability, functional design details, appearance, maintenance and cost. Students could be invited to suggest other criteria, based on their experiences with these products. This lecture focused primarily on protection, comfort and functional fit; students could also be asked to discuss any of the other selection criteria in an assignment.

The importance of characterizing and then simulating potential exposures to assess protection was discussed, along with how to measure protection. As with many products the potential exposure for a barrier face covering must be simulated using some other means. This could lead to a discussion of how to characterize an exposure, using research results. Ensuring that a particular challenge in a standard produces a range of performance among products was also discussed, along with trade-offs between protection and other criteria, such as comfort. To further illustrate this point, results from field experiments in which temperatures and heat fluxes are measured in fires are compared to the exposures used in standard tests for protective clothing.

Factors that influence comfort were outlined, including breathability, thermal and sensory comfort, and moisture management. As many students will have had experience wearing these products for an extended time, they could be invited to note any features that they found to be important. While some aspects of comfort are best assessed by wear studies and feedback from consumers, breathability can be evaluated using the pressure difference across a face covering. Moisture management can be evaluated using tests that measure water resistance and water vapour permeability. Resistance to liquids can play a role in protection - students could also be made aware of hazards that involve the impact of a stream of blood or other bodily fluids and how standards for these products evaluate this aspect of performance. The level of activity is also important; a product may be very comfortable while sitting at a desk, but not while exercising in a gym.

Functional fit was discussed, including leakage, sizing, methods to adjust the face covering and interactions with 
other components. Many students have the experience of having their glasses fog up while using a mask. While this may not be hazardous in itself, it does provide some indication of the seal between a product and user's face, which will also impact protection. Methods that can be used to size products to fit a range of users could be discussed, along with ways in which the product can be fastened (e.g., ear loops and ties) and adjusted. As with comfort, the importance of considering the level of activity is important.

The process used to develop the new ASTM F3502 standard was then described [9]. As with many standards organizations, ASTM has made this and other COVID-19related standards available for free, which is highlighted to the students (a quiz question also asks them to find the cost of another standard to provide an indication of the price of these documents). Design requirements in the specification were then discussed in detail, along with how these will help to meet the comfort and fit criteria. Performance requirements were outlined, along with how the conditioning of products before testing $\left(38 \pm 2.5^{\circ} \mathrm{C}, 85 \pm\right.$ $5 \% \mathrm{RH}$ ) differs from what is typically done in textile testing, in order to simulate the environment around the user's nose and mouth. Optional performance test results that manufacturers may choose to report were also mentioned, including bacterial filtration efficiency [7].

A discussion of the standards used to specify/regulate these products exposes students to methods used to consider health and safety risks in design. This topic can also be linked to a number of other graduate attributes [12]. The importance of involving a multi-disciplinary team is illustrated, along with the impact engineers can have through their involvement in these teams. The importance of understanding limitations of engineering tools and other methods used in design is emphasized, along with the role of engineers in protecting the public. The importance of communicating clear instructions to users to increase the effectiveness of these products can be discussed. Standards that result in the development of products that provide an improved level of protection will also reduce the use of disposable masks, which impacts the environment. Many engineers have become involved in developing products in response to the pandemic, and their ability to quickly develop the knowledge and skills necessary to design these products is an excellent illustration of the importance of life-long learning in one's career.

\section{REMOTE DESIGN ASSIGNMENTS AND ProjeCTS - Design OF TEST Methods}

The design of standard and non-standard tests was discussed in the lecture. The use of test standards as a starting point for experimental design was outlined, along with determining required data. For example researchers may require a larger number of thermocouples in a room than specified in a standard test when validating a CFD model of airflow in the space.

While the author's only contribution to ME 229 in 2020-21 was a codes and standards lecture, a short project based on the design of quantitative screening tests for candidate fabrics for barrier face coverings was also developed as an illustration for the lecture. While students did not have an opportunity to complete the project this year, it was designed so that it could be conducted at home using readily available materials, and therefore could be used as a remote design project or assignment in the future. Modified versions could be used for in-person activities (ME 229 includes a weekly lab period). Equipment was assembled at home by the author and used to test fabrics that could be used to make barrier face coverings.

The aim was to duplicate what students would have available to them at home, with minimal extra purchases. In this case, students could use measuring spoons, glasses, funnels, and plastic containers that would normally be recycled. This approach would also allow inexpensive kits to be assembled for students to complete labs or projects at home, as been done in other courses (e.g., [23]). Students could use fabrics already available in the home, or a set of fabric specimens could be distributed to students. The latter approach would allow a class to conduct a round-robin test program in the same fashion as those used to develop precision-and-bias statements in standards, which provide an indication of expected variability in test results.

As an example of the ideation phase, some qualitative and quantitative methods that have appeared since the spring of 2020 to help the public choose materials or commercials products were discussed. These include evaluating the water resistance of fabrics by observing whether a drop of water beads on the surface or immediately soaks into the fabric [22], or observing whether a spray of water is absorbed by a fabric or penetrates through [16]. Examples from one website that outlines qualitative and quantitative methods to evaluate the water resistance, breathability, filtration and durability of fabrics were presented [22]. Some aspects of performance are easier to measure than others. While, as noted above, filtration efficiency is evaluated in the new ASTM F3502 specification [9], these tests would be difficult to simulate in the home. However, information can be found on the range of particle sizes in common products, including sugar, flour and corn starch [22].

Tests that evaluate resistance to liquids are considerably easier to simulate, so this was the focus of the project. Standard tests used to assess protective clothing and equipment for resistance to water and other fluids were summarized, along with a project the author was involved in to measure the protection that flame resistant fabrics offer against splashes of hot water in an industrial 
setting [17]. The process used to develop a new test methods based on standard tests was briefly outlined. Athome versions of two standards were then shown to the students. Figure 1 shows an apparatus that would be similar to AATTC 42 [3], which is used to evaluate the rain penetration of fabrics and the barrier performance of protective fabrics and drapes. The weight of a piece of blotter paper placed behind the fabric is measured before and after a known quantity of water is poured through the funnel. A similar apparatus is also used in ASTM F2701 [8], but in this standard fabrics are rated on the basis of predicted burn injury to skin located underneath the fabric based on heat flux measurements. This test method was later modified in a graduate research project to include more test sensors, and flow patterns on the surface of the fabrics were studied [17]. The apparatus in Figure 1 could also be modified to evaluate the water resistance of the fabric when subjected to a water spray.

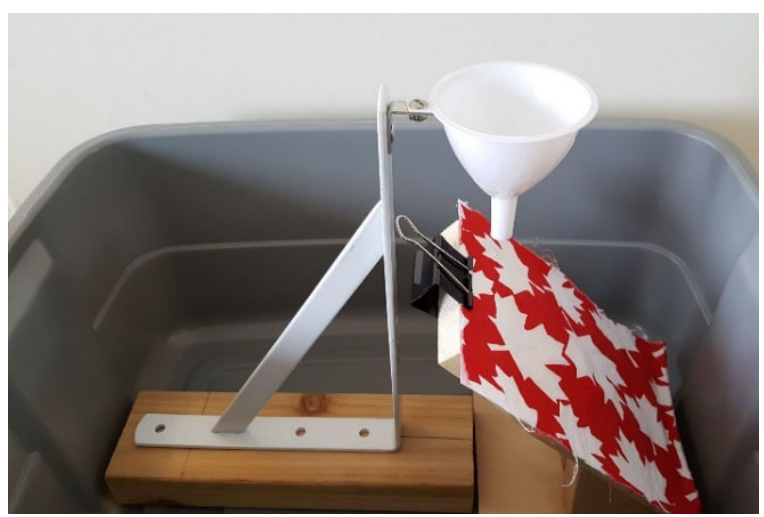

Fig. 1. Fabric test apparatus based on Gholameza [17], AATTC 42 [3] and ASTM F2701 [8].

Figure 2 shows another apparatus, which could be used by students to measure the fractions of the water hitting the surface of the fabric that are repelled, absorbed or transmitted. This is similar to one of the suggested screening tests [22], but in this case the fabric would be subjected to a flow of water from a funnel, rather than placing liquid onto the surface of the fabric. A scale with a resolution of $0.1 \mathrm{~g}$ may need to be purchased (about $\$ 15$ online) to weigh the fabric(s), cup and overflow basin before and after the test, as many scales used in home have a resolution of $1.0 \mathrm{~g}$. Otherwise, most of the other components should be available within the home (or could be included in a kit for students). The fabric is placed over a glass and held tightly in place using elastics. Approximately $10 \mathrm{~g}$ of water is poured into the funnel and the fabric, glass and catch basin are all weighted $60 \mathrm{~s}$ after the flow of water finishes. The fractions of the water that are repelled by the fabric (i.e., remaining on the surface of the fabric or in the catch basin), absorbed by the fabric and in the glass (i.e., transmitted) are then determined.

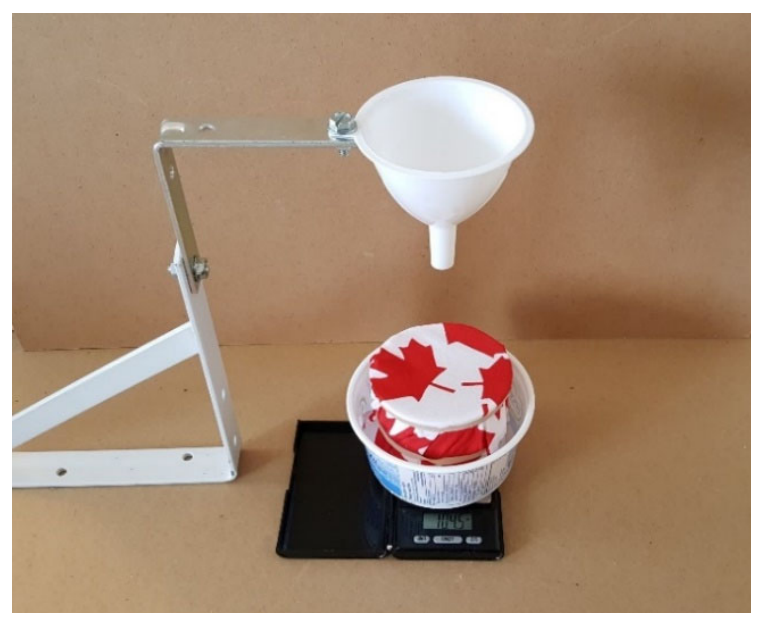

Fig. 2. Fabric test apparatus based on AATTC 42 [3].

As a demonstration, Figure 3 shows results obtained using the apparatus in Figure 2 for four $100 \%$ cotton woven fabrics $(\mathrm{BB}, \mathrm{CH}, \mathrm{MR}, \mathrm{NT})$ and a flannel fabric (PF) purchased from retailers. A pleated disposable mask (DM), a fabric taken from a cotton t-shirt (WT) and a single layer of polypropylene (PP) were also tested. The results indicated that while all of the woven fabrics had the same fibre content, some were able to repel a larger fraction of water, which resulted in less water transmission through the fabric. The ability of the flannel (PF) to absorb water was also demonstrated. While results in Figure 3 are based on measurements made after $60 \mathrm{~s}$, it was noted that in some cases water was not absorbed or transmitted through the fabrics until $30 \mathrm{~s}$ or more had elapsed. On the other hand the t-shirt material (WT) quickly absorbed the water, which was then transmitted to the glass. Both the disposable mask and the polypropylene repelled practically all of the water.

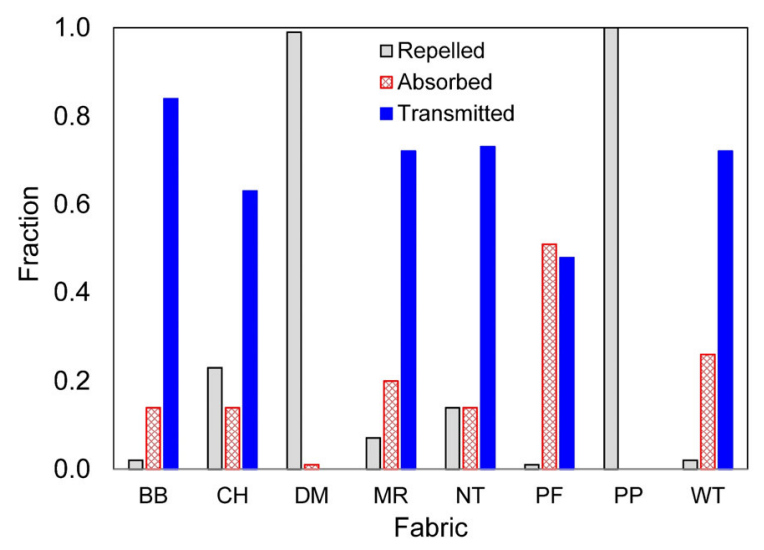

Fig. 3. Fractions of water repelled, absorbed and transmitted by various fabrics (woven cotton: $\mathrm{BB}, \mathrm{CH}, \mathrm{MR}$, NT, fleece: PF), a t-shirt (WT), a pleated disposable mask (DM) and a single layer of polypropylene (PP). 
Figure 4 shows results of tests of systems consisting of an outer layer made of one of the cotton fabrics shown in Figure 3, combined with an inner layer of another $100 \%$ cotton, woven fabric (GC). These were tested with and without a middle layer of polypropylene or flannel. The fraction absorbed shown in Figure 4 is based on the total amount of water absorbed by all layers of the fabric system. The disposable mask is shown again for comparison. Adding a middle layer eliminated practically all transmission of water. When polypropylene is added, it significantly improved the ability for the system to repel water. When flannel is added as a filter, it increased the amount of water absorbed in the system, and reduced the amount of water repelled.

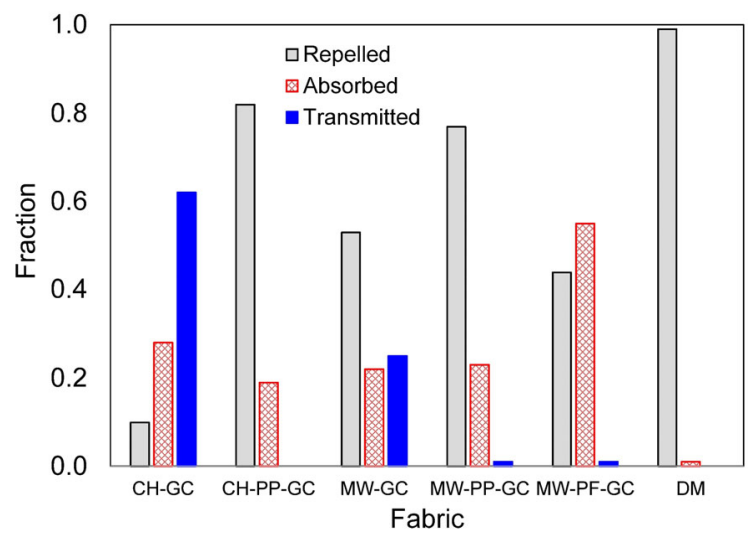

Fig. 4. Fractions of water that were repelled, absorbed and transmitted by double and triple-layer textile systems that include two different outer layers $(\mathrm{CH}, \mathrm{MW})$, the same inner layer (GC), with and without a polypropylene (PP) or

flannel (PF) layer in the middle, as well as a pleated disposable mask (DM).

Students could complete different types of analysis to support their designs, or to investigate changes in the test methods. Estimated impact velocities of liquids from test standards or research papers could be compared to the velocities in the test, which would inform the height of the funnel (the height for this demonstration was based solely on the location of holes in the brackets shown in Figures 1 and 2). The effect of this height on results for several fabrics could be examined. The total amount of water and the duration that it is allowed to sit on the surface of the fabric could also be investigated. These types of studies could also illustrate the importance of selecting test conditions that simulate a particular hazard, are repeatable, and differentiate candidate materials, which are important aspects of the design of standard tests.

Conditioning plays an important role in standard textile tests. A simple conditioning chamber could be built by students using a re-sealable container and an open dish containing a saturated solution of table salt. At room temperature this would be expected to produce a relative humidity of approximately $75 \%$ in the air within the sealed container [5]. Fabric performance could be compared at this humidity and at the ambient humidity in the home.

Another option would be have students use a spray, rather than using a flow of liquid. Students could refer to the literature for information on typical velocities and amounts of moisture in a cough or sneeze. Two pieces of equipment that could be used in the home would be spray bottles or metered delivery systems for saline sprays. Students could use their scale to determine the amount of liquid delivered by weighing a fabric before and after a number of sprays. This may also require designing a different system to hold the fabric(s) and a piece of paper behind the test specimen.

Other data characterizing the fabrics (e.g., permeability, scanning electron microscope images) could be provided to students to aid in discussion of test results. Some characterization of the fabrics could also be done by the students themselves (e.g., determining thread counts using digital images taken with a camera). While this project (and classroom discussion) focused on tests to measure liquid resistance, students could take a similar approach to develop methods to evaluate other aspects of performance, such as breathability.

\section{Conclusions}

This paper has outlined the importance of teaching students about the development of test standards used in engineering. Understanding the history of key standards will assist students in identifying potential limitations of standards used in design. The process of developing standard test methods for non-medical masks can be used as a case study in design courses, given students' experience with these products over the course of the pandemic. The design of test methods was also discussed. This topic leads itself to online projects and assignments that students could complete remotely. Data was presented from one example of a water repellency test that students could build at home using readily-available materials. While this project is framed in terms of non-medical masks, it could also be used to evaluate materials for outdoor performance clothing or other products, where water repellency is required.

\section{Acknowledgements}

Financial support from the University of Saskatchewan is gratefully acknowledged. 


\section{References}

[1] 42 CFR Part 84, Approval of respiratory protective devices. Available as of May 2, 2021, from https://ecfr.federalregister.gov/current/title-42/chapterI/subchapter-G/part-84?toc $=1$

[2] AATCC M14-2020, Guidance and considerations for general purpose textile face coverings: adult. American Association of Textile Chemists and Colorists, Research Triangle Park, NC, 2020.

[3] AATCC TM42, Test method for water resistance: impact penetration. American Association of Textile Chemists and Colorists, Research Triangle Park, NC, 2017.

[4] AFNOR Spec S76-001, Barrier masks: guide to minimum requirements, methods of testing, making and use. Association Française de Normalisation, Saint-Denis, France, 2020.

[5] ASTM E104-20a, Standard practice for maintaining constant relative humidity by means of aqueous solutions. ASTM International, West Conshohocken, PA, 2020.

[6] ASTM F2100, Standard specification for performance of materials used in medical face masks. ASTM International, West Conshohocken, PA, 2020.

[7] ASTM F2101, Standard test method for evaluating the bacterial filtration efficiency (BFE) of medical face mask materials, using a biological aerosol of staphylococcus aureus. ASTM International, West Conshohocken, PA, 2019.

[8] ASTM F2701-08, Standard test method for evaluating heat transfer through materials for protective clothing upon contact with a hot liquid splash. ASTM International, West Conshohocken, PA, 2008.

[9] ASTM F3502, Standard specification for barrier face coverings. ASTM International, West Conshohocken, PA, 2021.

[10] ASTM International, ASTM Compass. Available as of May 2, 2021, from

https://www.astm.org/Standard/enterprise-compass.html

[11] ASTM International, For professors: standards education: a lifelong learning foundation. Available as of May 2, 2021, from https://www.astm.org/studentmember/For Professors.ht $\underline{\mathrm{ml}}$

[12] Canadian Engineering Accreditation Board, 2020 Accreditation criteria and procedures. Engineers Canada, Ottawa, ON, 2020.

[13] Center for Disease Control, Use masks to slow the spread of COVID-19. Available as of May 2, 2021, from https://www.cdc.gov/coronavirus/2019-ncov/preventgetting-sick/diy-cloth-face-coverings.html
[14] CSA Z94.4-18, Selection, use and care of respirators, CSA Group, Toronto, ON, 2018.

[15] CWA 17553, CEN workshop agreement: community face coverings - guide to minimum requirements, methods of testing and use. European Committee for Standardization, Brussels, Belgium, 2020.

[16] Georgia Institute of Technology, Testing a fabric's suitability for cloth masks. Available as of May 2, 2021, from https://sites.gatech.edu/rapid-response/face-maskfabric-test/

[17] F. Gholamreza, Performance of thermal protective clothing upon exposure to hot liquid splash, $\mathrm{Ph} . \mathrm{D}$. dissertation, University of Alberta, Edmonton, AB, 2018

[18] Government of Canada, COVID-19 medical masks and respirators: how to get authorization. Available as of May 2, 2021, from https://www.canada.ca/en/healthcanada/services/drugs-health-products/covid19industry/medical-devices/personal-protectiveequipment/medical-masks-respirators/authorization.html

[19] Government of Canada, Non-medical masks: sew and nosew instructions. Available as of May 2, 2021, from https://www.canada.ca/en/publichealth/services/diseases/2019-novel-coronavirusinfection/prevention-risks/sew-no-sew-instructions-nonmedical-masks-face-coverings.html

[20] Johns Hopkins Medicine, Instructions on how to make a mask. Available as of May 2, 2021 from https://www.hopkinsmedicine.org/coronavirus/ docume nts/INF2003076 VW_HandSewn\%20Mask\%20instructions-1.pdf

[21] S. Kaplan, "How effective is your mask? You may know soon," New York Times, December 19, 2020, p. A10.

[22] MakerMask: Science-based mask information. Available as of May 2, 2021, from https://makermask.org/

[23] N. Samson, "Hands-on learning at home," University Affairs, Vol. 61, No. 5, November/December, 2020, p. 7.

[24] The Fabric Patch, Face Masks. Available as of May 2, 2021, from https:/www.fabricpatch.net/face-masks-forcovid-19-relief.htm

[25] P. Tronville and R. Rivers, "New methods for testing air filter performance," ASHRAE Journal, Vol. 58, No. 5, May, 2016, pp. 14-25.

[26] UnityPoint Health, Instructional video for sewing the Olson mask (COVID-19). Available as of May 2, 2021 from https://www.youtube.com/watch?v=ZnVk12sFRkY 